\title{
Letter to Editor: Volunteer Caregivers' Empowerment for the Care of COVID-19 Patients: An Experience in Iran
}

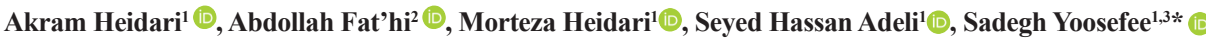 \\ 1. Spiritual Health Research Center, Qom University of Medical Sciences, Qom, Iran. \\ 2. Department of Philosophy, Imam Khomeini Education and Research Institute, Qom, Iran. \\ 3. Neuroscience Research Center, Qom University of Medical Sciences, Qom, Iran.
}

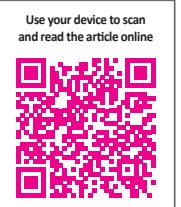

Citation: Heidari, A., Fat'hi, A., Heidari, M., Adeli, S. H., \& Yoosefee, S. (2020).Volunteer Caregivers' Empowerment for the Care of COVID-19 Patients: An Experience in Iran. Basic and Clinical Neuroscience, 11(3.Covid19), 247-250. http://dx.doi org/10.32598/bcn.11.covid19.606.4

http://dx.doi.org/10.32598/ben.11.covid19.606.4

\section{Dear Editor}

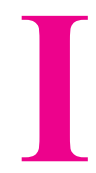

ranian Ministry of Health officially declared the two positive cases of Coronavirus Disease 2019 (COVID-19) on February 19, 2020 (National Committee on COVID-19 Epidemiology, 2020). Since then, the total number of confirmed cases reported by the World Health Organization has raised to 96448 cases until May 3, 2020 (World Health Organization, 2020). To manage and control the epidemic, the Emergency Operation Center (EOC) was organized with different working groups. Considering the mental health of the public, it seemed that some groups were more vulnerable to serious psychosocial consequences of the COVID-19 pandemic, including those in contact with the virus, patients with mental health problems, health care providers and professionals, and finally, public people exposed to the news through numerous media channels (Fiorillo, \& Gorwood, 2020; Hosseinzadeh-Shanjani, Hajimiri, Rostami, Ramazani, \& Dadashi, 2020).

In such circumstances, a holistic approach to the human being, as well as considering all physical, mental, social, and spiritual dimensions of the health and competent staff to deliver such services are necessary (Heidari, Heidari, \& Yoosefee, 2020; Grover, Dua, Sahoo, Mehra, Nehra, \&
Chakrabarti, 2020). However, providing holistic care for patients was difficult because of not only the increasing number of patients admitted to the hospitals and the lack of adequate health care personnel but also due to the complications of the disease. The health care providers' main concern about physical health can create a working group focusing specifically on the neglected aspects of health in an epidemic situation through alternative ways. Several university faculty members, psychologists, clerics, and volunteer agents gathered together in this working group and decided to take rapid actions to empower the volunteer caregivers to provide mental and spiritual care for the patients besides their companionship tasks. The empowerment process was conducted according to the flowchart shown in Figure 1, including the following steps:

- Assessing needs, setting educational goals, and developing a curriculum

- Identifying the most experienced professors in all fields

- Teaching the courses while videotaping and preparing electronic content

- Publishing the media 


\section{Process of empowering volunteer caregivers}

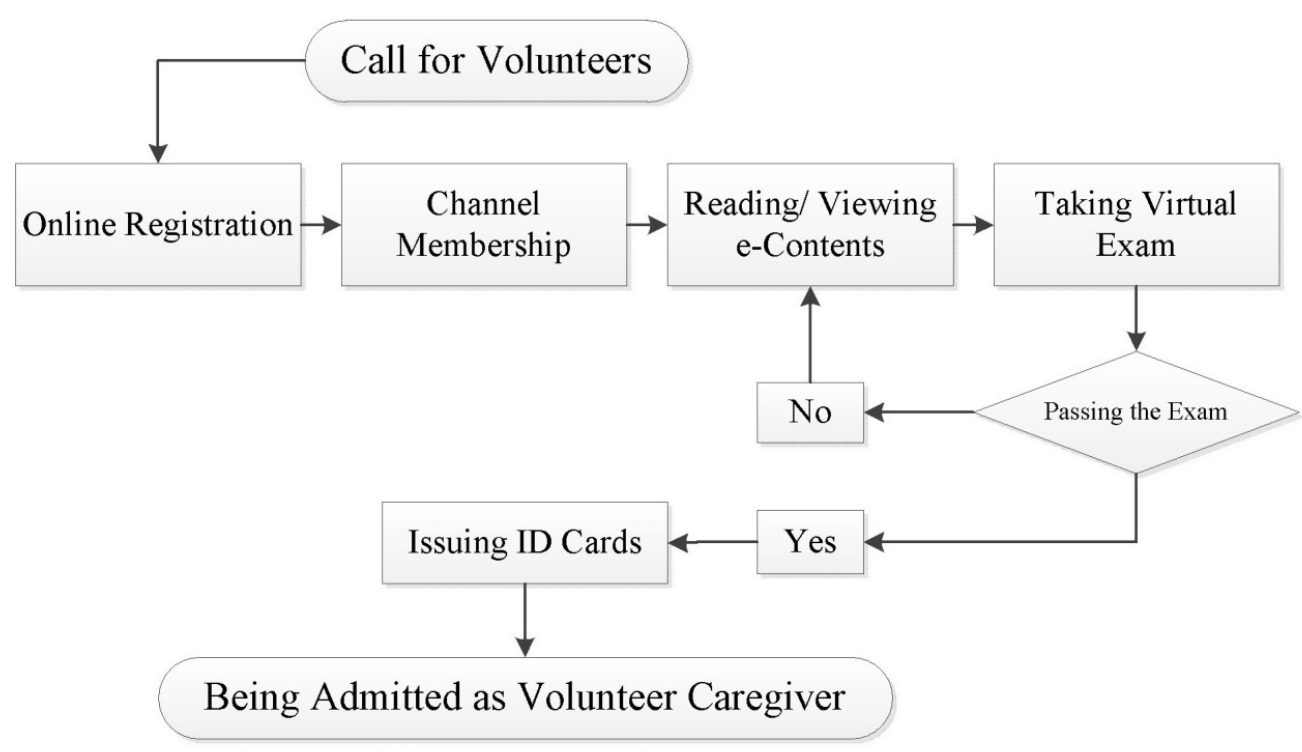

Figure 1. The flowchart of the volunteer caregivers' empowerment process

As shown, the registered volunteers received the content in both audiovisual and text formats. They were required to take an online exam after taking part in the courses before being accepted as caregivers. Also, 25 online exams were held from early March to the end of April 2020, during which 675 people participated and 325 passed the exams. Those passing the exam received ID cards and were introduced to the officer in charge of distributing them among hospitals according to the needs. Only those with cards were allowed to be recruited as volunteer caregivers in the hospitals.

Different topics were included in the course as compulsory and optional contents. The course focused on basics and key practical points of each topic, including:

- Personal protection and self-care against coronavirus;

- A brief review of medical terminology;

- Effective communication skills;

- Counseling skills;

- Psychological harms of COVID-19;

- Psychology of health and disease;

- Spiritual screening process;
- Spiritual care at the bedside;

- Empathy;

- Stress management;

- Hope development;

- Spiritual resilience.

Since the quality of health care delivery in critical situations is very important and a step towards whole-person care, it seems that the above-mentioned experience is applicable everywhere confronting similar problems. It is obvious that public participation is the main prerequisite of its implementation.

\section{Ethical Considerations}

Compliance with ethical guidelines

All ethical principles were considered in this article

Funding

There was no funding for the research reported in the present article. 


\section{Authors' contributions}

Drafted the letter: Akram Heidari, Sadegh Yoosefee. Provided critical input and edited the manuscript: Abdollah Fat'hi, Morteza Heidari, Seyed Hassan Adeli; Read and approved the final manuscript: All authors.

\section{Conflict of interest}

The authors declared that they have no competing interests.

\section{Acknowledgments}

We appreciate all the colleagues in Qom University of Medical Sciences and the volunteers' agents who contributed in this work.

\section{References:}

Mounesan L, Eybpoosh S, Haghdoost A, Moradi G, Mostafavi E. Is reporting many cases of COVID-19 in Iran due to strength or weakness of Iran's health system? Iranian Journal of Microbiology, 2020, 12(2), 73. https://ijm.tums.ac.ir/index.php/ijm/ article/view/2556

World Health Organization. (2020). Coronavirus Disease (COVID-2019). Available from: https://www.who.int/docs/ default-source/coronaviruse/situation-reports/20200503covid-19-sitrep-104.pdf?sfvrsn=53328f46_2

Fiorillo, A., \& Gorwood, P. The consequences of the COVID-19 pandemic on mental health and implications for clinical practice. European Psychiatry, 63(1),e32 [DOI:10.1192/j.eurpp sy.2020.35] [PMID] [PMCID]

Heidari, M., Heidari, A., \& Yoosefee, S. (2020). COVID-19 Pandemic and the Necessity of Spiritual Care. Iranian Journal of Psychiatry, 15(3), 262-263.

Hosseinzadeh-Shanjani, Z., Hajimiri, K., Rostami, B., Ramazani, S., \& Dadashi, M. (2020). Stress, anxiety, and depression levels among healthcare staff during the COVID-19 Epidemic. Basic and Clinical Neuroscience, 11(2), 163-70. [DOI:10.32598/bcn.11. covid19.651.4]

Grover, S., Dua, D., Sahoo, S., Mehra, A., Nehra, R., \& Chakrabarti, S. (2020). Why all COVID-19 hospitals should have mental health professionals: The importance of mental health in a worldwide crisis! Asian Journal of Psychiatry, 51, 102147. [DOI:10.1016/j.ajp.2020.102147] [PMID] [PMCID] 
This Page Intentionally Left Blank 